\title{
Knowledge of cardiopulmonary resuscitation among Greek physiotherapists
}

\author{
Garyfallia Pepera $^{1}$, Efstratios Xanthos ${ }^{2}$, Andreas Lilios ${ }^{2}$, Theodoros Xanthos ${ }^{2,3}$ \\ ${ }^{1}$ Department of Physiotherapy, University of Thessaly, Lamia, Greece; ${ }^{2}$ Postgraduate Study Program (MSc) \\ “Cardiopulmonary Resuscitation”, National and Kapodestrian University of Athens, Greece; ${ }^{3}$ School of Medicine, \\ European University Cyprus, Nicosia, Cyprus
}

\begin{abstract}
High quality cardiopulmonary resuscitation (CPR) is crucial for influencing survival from cardiac arrest. Healthcare professionals are expected to know how to perform CPR as they may encounter emergency situations during their work. Physiotherapists, who use exercise as a therapeutic approach, should have good knowledge and skills in CPR not only to cope with possible adverse cardiac events during exercise but also because a widespread CPR application and early defibrillation can greatly reduce mortality due to heart attack. The aim of this study is to investigate knowledge of Greek physiotherapists in European Resuscitation Council guidelines for resuscitation. A secondary aim of this study was to assess and compare the knowledge score between those with and without previous training and/or lower self-confidence in CPR skills. Three hundred and fifty Greek physiotherapists who were working in hospitals and rehabilitation centres (face-to-face and e-mail contact) were randomly selected to complete an anonymous questionnaire containing demographic questions, CPR experience questions, and ten theoretical knowledge questions, based on European Resuscitation Council guidelines for resuscitation. The response ratio was $63 \%(n=220$ physiotherapists). Respondents' total mean score for the theoretic
\end{abstract}

Correspondence: Garyfallia Pepera, Department of Physiotherapy, University of Thessaly, $3^{\text {rd }} \mathrm{km}$ of Old National Road, 35100 Lamia, Greece. Tel.+30.2231060207. E-mail: gpepera@uth.gr

Key words: Cardiopulmonary resuscitation; physical therapists; cardiac arrest; theoretical knowledge; European Resuscitation Council Guidelines.

Ethics: All procedures were approved by The University Ethics Committee (University of Athens, Medical School) and conformed to the declaration of Helsinki guidelines for research with human subjects.

Conflict of interest: the authors declare no conflict of interest.

Received for publication: 27 June 2019.

Accepted for publication: 16 October 2019.

${ }^{\circ}$ Copyright: the Author(s), 2019

Licensee PAGEPress, Italy

Monaldi Archives for Chest Disease 2019; 89:1124

doi: 10.4081/monaldi.2019.1124

This article is distributed under the terms of the Creative Commons Attribution Noncommercial License (by-nc 4.0) which permits any noncommercial use, distribution, and reproduction in any medium, provided the original author(s) and source are credited. knowledge questions was $4.1 \pm 2$ (range $1-10$ ); $21.4 \%$ of the respondents had participated in a CPR course, while only $0.9 \%$ had previous experience in CPR performance. The group of respondents who had attended a CPR course had a significantly higher score in CPR knowledge questions and higher confidence score $(p<0.01)$. Moreover, the physiotherapists who attended refresher courses in CPR in the workplace scored significantly higher $(p<0.01)$. Our results indicate that Greek physiotherapists have knowledge gaps in the European Resuscitation Council guidelines for resuscitation. The percentage of Greek physiotherapists who had CPR certification and recertification was low, thus the CPR training should be mandatory for all working physiotherapists.

\section{Introduction}

Cardiac arrest constitutes a major public health issue and accounts for more than 400,000 deaths annually. Cardiac arrest can be associated with a shockable or non-shockable rhythm [1]. Survival rates after sudden cardiac arrest have improved in the past decades (6.4\% after out-of-hospital cardiac arrest and 18\% after in-hospital cardiac arrest among adults) but remain a major public health concern [2]. Survival following cardiac arrest depends on early diagnosis, the quality of cardiopulmonary resuscitation (CPR) and early defibrillation [3]. Despite the high quality and effectiveness of CPR when relevant guidelines are applied, the percentage of the correct application of chest compressions (depth and frequency are $39 \%$ and $56 \%$ respectively) and the compression-to-ventilation ratio remains suboptimal [4].

The International Resuscitation Organizations and the European Resuscitation Council highlight the importance of training in delivering high-quality CPR [5] and support that knowledge of CPR wears off over time [6,7], due to either a lack of CPR practice, or an absence of systematic refresher training by healthcare professionals [6]. Training in CPR should be repeated nearly every seven months so that the quality of CPR can be maintained at the required high levels [8]. Apart from cardiac arrests happening inhospital, fatal or non-fatal cardiovascular events take place during physical activities. Despite exercise benefits [9], this may trigger cardiac infarction or arrest in patients with coronary disease $[10,11]$. It is also well-established that during exercise the risk of sudden cardiac arrest is increased [10,11]. During rehabilitation, cardiovascular event rates range from 12.3 to 37.4 per million patient/hours of exercise [11-13]. Fatal cardiac events are reported more rarely and ranged from 8.6 per million patient hours of exercise in 1978 to zero in the present day [13]. Incidence of infarction and arrest during supervised exercise is extremely low. Supervised exercise testing and training in cardiovascular disease patients are accompanied only by minor cardiovascular events [14]. The same 
research reports no events related to hospitalization, syncope episodes or fatality [14].

Many studies have examined the competence of medical and health professionals in CPR delivery [15-18]. Physiotherapist is a healthcare professional who might work in the context of acute and critical care [19], and with managing who should be able to treat heart diseases even during exercise. To the best of our knowledge, no other studies have investigated physiotherapist's knowledge in CPR. This study aims to investigate knowledge of Greek physiotherapists in European Resuscitation Council guidelines for resuscitation. A secondary aim of this study was to assess and compare the knowledge score between those with and without previous training and/ or had lower self-confidence in CPR skills.

\section{Patients and Methods}

\section{Study design and data collection}

An anonymous questionnaire [16] was piloted in 10 certified resuscitation instructors from training centres in Athens and by 20 physiotherapists (not included in the results of the main study). The questionnaire was initially evaluated in terms of quality, clarity, and comprehensiveness of the chosen questions by 10 certified resuscitation instructors who were free to suggest amendments. The instructors found the questionnaire clear and no amendments were required. Then the questionnaire was tested on a small randomly selected sample of 20 physiotherapists working in hospitals and private practices. The questionnaire's reliability was assessed by using a test-retest procedure (Intraclass correlation coefficient, ICC) within a week period [16]. All the physiotherapists were asked to fill out the questionnaire 1 week later. The mean level of answer agreement was $95 \%$ between the two measurements (ICC range, $90-100 \%$; $\mathrm{SD}, 4 \%$ ).

\section{Questionnaire}

The questionnaire comprised three sections with 20 questions in total. The first section included six items regarding demographic data (age, gender, level of education, training in CPR), the second section comprised of 4 closed questions relating to the participants' experience and confidence in CPR self-reported in a Likert scale (1, very low; 2 , low; 3 , moderate; 4,high; 5,very high). The third section included ten multiple-choice questions relating to CPR, which were based on European Resuscitation Council Guidelines for Resuscitation [20]. Each question had only one correct answer out of four choices and there was no negative marking. In the third section, a marking system was used to assess knowledge of Greek physiotherapists in European Resuscitation Council guidelines for resuscitation, each correct answer being allocated one point, zero being the lowest mark and ten being the highest.

\section{Sample}

This study was conducted in various hospitals and private rehabilitation centres in Athens, Greece. The total number of physiotherapists in Greece is 7000. A random 5\% sample was considered representative of the population. Greek physiotherapists who were registered in the Pan-Hellenic Physiotherapy Association registry (the official body of registered physiotherapists within Greece working either in the inpatient or outpatient departments in Greece) were randomly selected using a random number generator, and 350 participated in the survey. Physiotherapists were invited to participate via face-to-face or e-mail contact.

\section{Ethical approval}

Ethical approval was granted by the local Research Ethics Committee and the director of research at each hospital. Questionnaires were returned anonymously and kept in a safe place.

\section{Statistical analysis}

Simple frequencies and proportions were calculated. Continuous variables are presented as means ( \pm standard deviation). Student $t$ test was used to compare the difference for continuous data. Subjects were grouped for age, sex, specialization, training in CPR, education on changes in CPR, experience, and confidence in applying CPR. Chi-square test was used to examine correlation between correct answers in CPR related questions and categorical variables, like training in CPR and confidence in CPR delivery. Statistical significance was set at $\mathrm{p}<0.05$ The Statistical Package for Social Sciences (SPSS ver. 19) was used for all analyses.

\section{Results}

The response ratio was $63 \%(n=220$ physiotherapists $)$ and demographic characteristics are shown in Table 1 . None of these respondents withdrew from the survey. Respondents' total mean score for the theoretic knowledge questions was $4.1 \pm 2$.

The mean percentage of respondents whose answers adhered to European Resuscitation Council guidelines for resuscitation are presented in Table 2. Question \#10 'Principles of chest compressions' had the highest success rate; $59.1 \%$ of the participating physiotherapists responded correctly and the question 5 'Initial CPR action when an adult suddenly collapses' had the lowest success rate; $17.3 \%$ of the participating physiotherapists responded correctly.

Regarding previous CPR personal resuscitation, $0.9 \%$ of the study group reported performing CPR on cardiac arrest victims, while $21.4 \%$ of them had attended a CPR course. There was statistically significant difference in CPR knowledge scores between respondents who had received CPR training, and those who had not [6.4 (1.9) vs 3.4 (1.5) points, respectively; $\mathrm{p}=0.01$ ].

Chi-square test revealed a significant correlation between responders who attained low scores in CPR questionnaire and those who had not been trained in CPR $\left(\chi^{2}(1, n=220)=60.84\right.$; $\mathrm{p}<0.001) ; 65.9 \%$ of the respondents scored below 5 out of 10 .

The respondents' confidence in delivering CPR was significantly correlated with the final performance in the written questionnaire test $\left(\chi^{2}(1, n=220)=60.2, p<0.001\right) .71 \%$ of the respondents who scored less than 5 in CPR (theoretical knowledge questions) had very low confidence and none of those who scored less than 5 had very high confidence. In addition, 36\% of respondents who scored above 5 had low confidence and $20 \%$ of them had high confidence.

\section{Discussion}

Despite global organizations for CPR stressing the importance of the quality of CPR skills and training [16], the latter remain low [15,25-31]. Despite the acknowledged benefit of CPR skills in health professionals and the general public [15-18,24], no previous study has investigated the knowledge of CPR among physiotherapists. This study examined the knowledge of Greek physiotherapists in CPR delivery working both in private and public centres. 
Our study showed that although physiotherapists are expected to have skills and knowledge in order to resuscitate patient in an emergency, they have knowledge gaps in European Resuscitation Council guidelines for resuscitation. This confirms the results of other similar studies among different healthcare professionals [1517,31], even among cardiologists alone [15]. The knowledge of specialised life support among a group of Greek cardiologists was examined and it was reported that $75.9 \%$ of the cardiologists had performed chest compressions in the past, and found that participants who had been trained in CPR scored higher in theoretical CPR knowledge, and in specialised CPR, than untrained participants [15].

There is an evident lack of low level in knowledge of CPR among nurses [16]. Furthermore, another study showed that there is a significant difference $(\mathrm{p}<0.01)$ in healthcare professionals' (doctors and nurses) knowledge between those who have been trained in CPR delivery and those who have been not [17]. A team of researchers in Finland and Hungary, after evaluating the knowledge and competence of professional nurses and students in nursing schools, found only $53 \%$ of them had been trained in European Resuscitation Council guidelines for resuscitation. They also found $21 \%$ of the participants responded correctly regarding hand position in chest compressions, and $33 \%$ of them responded correctly regarding the compression-to-ventilation ratio [18]. These findings from previous studies underline the need for education in CPR performance and make it a mandatory component of all healthcare professions.

The knowledge of European Resuscitation Council guidelines for resuscitation in Greek physiotherapists, as measured by means of a closed-answer questionnaire, was in accordance with levels reported elsewhere [15-18,23]. Moreover, results imply a relative-

Table 1. Descriptive characteristics of the study group.

\begin{tabular}{ll} 
Characteristics & Values \\
Number of respondents & 220 \\
Age, years & $40 \pm 11$ years \\
\hline Sex (n, \%) & Males=106 (48.2\%) - Females=114 (51.8\%) \\
Professional degree & University \\
\hline Specialization & $1(0.5 \%)$ \\
Cardiovascular rehabilitation & $3(1.4 \%)$ \\
Musculoskeletal rehabilitation & $1(0.5 \%)$ \\
Pulmonary rehabilitation & $5(2.3 \%)$ \\
Neurological rehabilitation & $210(95.5 \%)$ \\
None & $47(21.4 \%)$ \\
CPR course attendance & $2(0.9 \%)$ \\
\hline Personal experience of performing CPR in adult & $14(6.4 \%)$ \\
Updated on changes in the CPR guidelines & $5(2.3 \%)$ \\
\hline Education on CPR changes in the workplace & $130(59.1 \%)$ \\
Confidence in performing resuscitation skills & $49(22.3 \%)$ \\
Very low & $21(9.5 \%)$ \\
Low & $19(8.6 \%)$ \\
Average & $1(0.5 \%)$ \\
High &
\end{tabular}

CPR, cardiopulmonary resuscitation.

Table 2. Percentage of correct answers of physiotherapist for each of the ten CPR questions separately.

According to European resuscitation guidelines for Resuscitation, what is the compression to ventilation ratio in an adult victim? What is the rate of chest compression per minute?

$10(50 \%) \quad 110(50 \%)$

What is the primary action in order to keep the airway open? $75(34 \%) \quad 145(66 \%)$

What is the depth of chest compressions to be achieved by the rescuer in order for the resuscitation to be effective? $99(45 \%) \quad 121(55 \%)$ What is the initial CPR action when an adult suddenly collapses? $64(29 \%) \quad 156(71 \%)$ What is the first action to rescue a conscious choking victim $37(17 \%) \quad 183(83 \%)$

What can we do before defibrillation? $119(54 \%) \quad 101(46 \%)$

What is the most common sign for the recognition of cardiac arrest? $99(45 \%) \quad 121(55 \%)$ $48(22 \%) \quad 172(78 \%)$

When we call the local emergency number for an ambulance what information should we provide?

$110(50 \%) \quad 110(50 \%)$

Principles of chest compressions (compression-decompression resuscitation)

$130(59 \%) \quad 90(41 \%)$

$\mathrm{CPR}$, cardiopulmonary resuscitation. 
ly poor theoretical background in CPR, which could be explained by the fact that $78.6 \%$ of the study group had not received relevant training. Previous studies among medical and paramedical staff have reported that the percentage of staff who did not receive prior CPR training ranged between 7\% [23] and 69\% [30].

Training in CPR is crucial in establishing a solid theoretical background and in applying effective defibrillation [32]. The former was observed among participants of the current study who had been trained in CPR and had more correct answers (83\% of participants scored above 5) as compared to those without CPR training ( $21 \%$ of participants scored over 5). The results agree with those of Xanthos et al. [16] and Passali et al. [17], but not with the results of Akpek et al. (2003) [22]: the only study which reported non-significant differences in overall score rates between participants with and without CPR training.

Our data showed that more than half $(51.8 \%)$ of respondents gave incorrect responses to CPR questions (compression to ventilation ratio), suggesting inadequate underpinning knowledge and training in CPR. False responses rate was considerably high, ranging from $77.3 \%$ [33] to $80 \%$ of the respondents [16]. Thus, the level of theoretical knowledge of physiotherapists participating in this study and their achievement in the testing part of the questionnaire is in accordance with those reported by other studies, and are regarded as inadequate. More than half of the respondents' physiotherapists (59.1\%) ranked their level in CPR knowledge as "low" in the relevant self-assessment question, which means that they know their knowledge and skills of providing CPR should be improved. These skills should be fundamental knowledge for all those physiotherapists who work at hospital and in rehabilitation centres. In another study [16], nurses showed major gaps in the CPR, even though their score was higher.

Moreover, trained and more self-confident physiotherapists did answer better in questions about CPR compared to those had not been trained and/or had lower self-confidence. This fact underlines the importance of training in achieving and maintaining a solid theoretical background in CPR. In turn, the latter is crucial since patient survival depends on time of early defibrillation and the quality of CPR delivery [3], as stressed out in the latest guidelines [16].

\section{Study limitations}

All physiotherapists included in our study were randomly selected and the response rate to the questionnaire was remarkably good, in consideration of the fact that multiple contacts impact on survey response rates [34]. However, lack of comparison between privately-practicing and in-hospital physiotherapists should be considered a limitation of the present study, as the latter - working in intensive care unit and other emergency units- are expected to actually have more qualifications in resuscitation. Also, differentiating among physiotherapists employed in coronary care units, intensive care units and in rehabilitation following cardiovascular disorders, as well as assessing practical skills in CPR are research parameters that would provide valuable information and inform further research, along with the results that this study already documented. Another limitation of the study is the absence of any possibility to compare the results of the questionnaire to other available tests, since the authors specifically created the evaluation questionnaire. Furthermore, the study examined physiotherapists' theoretical knowledge and no practical skills performance on resuscitation. Future studies should address this issue and evaluate competence in the practical application of CPR. Moreover, healthcare professionals in CPR knowledge and skills differ between countries. However, physiotherapists should be evaluated globally on performing good quality cardiopulmonary resuscitation.

\section{Conclusions}

Physiotherapists are expected to be knowledgeable in performing CPR. The results of this study indicate, however, that Greek physiotherapists' knowledge of the European resuscitation guidelines is low and does not meet the recommended level.

Few respondents had been trained in CPR, had been confident in its application, had been informed in their workplace in CPR advancements and scored high in the relevant test. Results indicate the need for physiotherapists to get better educated and stay informed on changes in CPR. Ideally, mandatory accreditation procedures in CPR competency should be set in place for all physiotherapists, regardless of their specific workplace, to ensure sufficient knowledge and quality in CPR delivery. Physiotherapists, as healthcare professionals, should be trained and periodically evaluated on performing good quality CPR.

\section{References}

1. Murakoshi N, Aonuma K. Epidemiology of arrhythmias and sudden cardiac death in Asia. Circulation 2013;77:2419-31.

2. Sidney S, Rosamond WD, Howard VJ, et al. The "heart disease and stroke statistics--2013 update" and the need for a national cardiovascular surveillance system. Circulation 2013;127:21-3.

3. Gwinnutt CL, Columb M, Harris R. Outcome after cardiac arrest in adults in UK hospitals: effect of the 1997 guidelines. Resuscitation 2000;47:125-35.

4. Brown TB, Dias JA, Saini D, et al. Relationship between knowledge of cardiopulmonary resuscitation guidelines and performance. Resuscitation 2006;69:253-61.

5. Chamberlain D, Hazinski M. Education in resuscitation. Resuscitation 2003;59:11-43.

6. Hamilton R. Nurses' knowledge and skill retention following cardiopulmonary resuscitation training: a review of the literature. J Adv Nurs 2005;51:288-97.

7. Smith K, Gilcreast D, Pierce K. Evaluation of staff's retention of ACLS and BLS skills. Resuscitation 2008;78:59-65.

8. Woollard M, Whitfield R, Newcombe RG, et al. Optimal refresher training intervals for AED and CPR skills: a randomised controlled trial. Resuscitation 2006;71:237-47.

9. Wardyn GG, Rennard SI, Brusnahan SK, et al. Effects of exercise on hematological parameters, circulating side population cells, and cytokines. Exp Hemat 2008;36:216-23.

10. Tofler G, Muller J. Triggering of acute cardiovascular disease and potential preventive strategies. Circulation 2006;114:1863-72.

11. Haskell W. Cardiovascular complications during exercise training of cardiac patients. Circulation 1978 57:920-4.

12. Friedewald V, Spence D. Sudden cardiac death associated with exercise: the risk-benefit issue. Am J Cardiol 1990;66:183-8.

13. Van Camp S, Peterson R. Cardiovascular complications of outpatient cardiac rehabilitation programs. JAMA 1986; 56:1160-3.

14. Pepera G, Sandercock, G. A pilot study to investigate the safety of exercise training and testing in cardiac rehabilitation patients. Br J Cardiol 2013;20:78.

15. Pantazopoulos I, Aggelina A, Barouxis D, Papapanagiotou P, Troupis G, Kotsiomitis E, et al. Cardiologists' knowledge of the 2005 American Heart Association Resuscitation Guidelines: The Athens Study. Heart Lung 2011;40:278-84.

16. Xanthos T, Akrivopoulou A, Pantazopoulos I, et al. Evaluation of nurses' theoretical knowledge in basic life support: a study 
in a district Greek hospital. Intern Emerg Nurs 2012;20:28-32.

17. Passali C, Pantazopoulos I, Dontas I, et al. Evaluation of nurses' and doctors' knowledge of basic \& advanced life support resuscitation guidelines. Nurse Educ Pract 2011;11:365-9.

18. Nyman J, Sihvonen M. Cardiopulmonary resuscitation skills in nurses and nursing students. Resuscitation 2000;47:179-84.

19. Stiller K. Physiotherapy in intensive care: an updated systematic review. Chest 2013;144:825-47.

20. Cirio S, Piaggi GC, De Mattia E, Nava S. Muscle retraining in ICU patients. Monaldi Arch Chest Dis. 2003;59:300-3.

21. Nolan JP, Soar J, Zideman DA, et al. European Resuscitation Council Guidelines for Resuscitation 2010 Section 1. Executive summary. Resuscitation 2010;81:1219-76.

22. Cohen J. Statistical power analysis for the behavioural sciences. New Jersey: Lawrence Erlbaum Associates; 1988.

23. Akpek E, Kayhan Z. Knowledge of basic life support: a pilot study of the Turkish population by Baskent University in Ankara. Resuscitation 2003;58:187-92.

24. Chandrasekaran S, Kumar S, Bhat SA, et al. Awareness of basic life support among medical, dental, nursing students and doctors. Indian J Anaesth 2010;54:121-6.

25. Chaudhari MS, Panchal NN, Kamat HV, Ganjiwale J. Knowledge of 2015 basic life support (BLS) guidelines among doctors and nursing staff of a rural based tertiary care hospital, in western India: current status and requirement. Indian J Anaesth 2017;4:193-7.

26. Majid A, Jamali M, Ashrafi MM, et al. Knowledge and attitude towards cardiopulmonary resuscitation among doctors of a tertiary care hospital in Karachi. Cureus 2019;11:e4182.

27. Myrianthefs P, Kalafati M, Lemonidou C, et al. Efficacy of CPR in a general, adult ICU. Resuscitation 2003 57:43-8.

28. Nambiar M, Nedungalaparambil NM, Aslesh OP- Is current training in basic and advanced cardiac life support (BLS \& ACLS) effective? A study of BLS \& ACLS knowledge amongst healthcare professionals of North-Kerala. World J Emerg Med 2016;7:263-9.

29. Osinaike B, Aderinto D, Oyebamiji E, et al. Evaluation of knowledge of doctors in a Nigrian tertiary hospital of CPR. Niger Med Pract 2007;52:16-8.

30. Roshana S, Batajoo K, Piryani R, Sharma M. Basic life support: knowledge and attitude of medical/paramedical professionals. World J Emerg Med 2012;3:141-5.

31. Kanstad BK, Nilsen SA, Fredriksen K. CPR knowledge and attitude to performing bystander CPR among secondary school students in Norway. Resuscitation 2011; 82:1053-9.

32. Kallestedt ML, Rosenblad A, Leppert J, et al. Hospital employees' theoretical knowledge on what to do in an in-hospital cardiac arrest. Scand J Trauma Resusc Emerg Med 2010;18:43.

33. Keenan M, Joubert G. A survey of nurse's basic life support knowledge and training at a tertiary hospital. Afric J Health Prof Educ 2009;1:3-7.

34. Fincham J. Response rates and responsiveness for surveys, standards, and the journal. Am J Pharm Educ 2008;72:43. 
